\title{
Percepção dos alunos de uma universidade pública sobre o Programa de Educação pelo Trabalho para a Saúde
}

\author{
Perception of students of a public university \\ on the Education Program for Work in Health
}

Anna Carolina Martins Pinto ${ }^{1}$

Isabela Viana Oliveira ${ }^{2}$

Ana Luiza Soares dos Santos ${ }^{2}$

Luiza Eunice Sá da Silva ${ }^{2}$

Gabriela da Silva Lourelli Izidoro ${ }^{2}$

Raquel de Deus Mendonça ${ }^{3}$

Aline Cristine Souza Lopes ${ }^{3}$

${ }^{1}$ Secretaria Municipal de Saúde de Belo Horizonte. Av. Gen. David Sarnoff 3113, Cidade Industrial. 32.210-110 Contagem MG. aline@efn.ufmg.br ${ }^{2}$ PET-Saúde, UFMG ${ }^{3}$ Escola de Enfermagem, Universidade Federal de Minas Gerais.

\begin{abstract}
This study sought to analyze the Education Program for Work in Health from the perspective of students at a public university in Belo Horizonte, State of Minas Gerais, in their first year and its impact on the students' qualification. It is a prospective cohort study with students who participated on this program, and the data was collected by a structured and self-applied questionnaire, during the sixth and tenth months of the program, with sociodemographic questions, methodology, aims and impact of the program. The majority reported that the program's proposed aims were attained, especially interdisciplinary work and academic research focused on the Unified Health System's needs. The program also contributed to training in research, teaching and extension work, and in the second evaluation, the students reported greater interaction with social equipment. It is considered that the program is an important strategy for human resources training in accordance with the Unified Health System's needs, by enabling the exercise of teamwork, communication, agility and creativity to deal with adverse situations, as well as the practice of the primary health care principles integrated in the community.

Key words Human resources training, Professional education in public health, Teaching care integration services, Primary Health Care, Unified Health System
\end{abstract}

Resumo Este estudo objetivou analisar, na perspectiva dos alunos, o Programa de Educação pelo Trabalho para a Saúde (PET-Saúde) de uma universidade pública de Belo Horizonte-MG em seu primeiro ano e seu impacto na formação dos discentes. Estudo de coorte prospectivo com os alunos participantes do PET-Saúde, com dados coletados por questionário estruturado e autoaplicado, aos seis e dez meses do programa, com questões sociodemográficas, de avaliação da metodologia, objetivos e impacto do programa. A maioria relatou que os objetivos propostos foram alcançados, destacando o trabalho interdisciplinar e a produção acadêmica voltada para as necessidades do Sistema Único de Saúde (SUS). O programa também contribuiu para a formação profissional nas áreas de pesquisa, ensino e extensão, sendo que na segunda avaliação, os discentes relataram maior interação com equipamentos sociais. Acredita-se que o PET-Saúde constitui importante estratégia de formação de recursos humanos conforme as necessidades do SUS, por possibilitar o exercício do trabalho em equipe, da comunicação, agilidade e criatividade para lidar com situações adversas, bem como a vivência dos princípios da Atenção Primária integrados à comunidade.

Palavras-chave Formação de recursos humanos, Educação profissional em saúde pública, Serviços de integração docente-assistencial, Atenção Primária à Saúde, Sistema Único de Saúde 


\section{Introdução}

A Constituição Federal prevê como uma das competências do Sistema Único de Saúde (SUS) ordenar a formação de Recursos Humanos (RH) e incrementar a área de atuação com desenvolvimento científico e tecnológico ${ }^{1}$. Neste sentido, a Lei ${ }^{\circ} 8080$, de 19 de setembro de 1990 define que a União, os Estados, o Distrito Federal e os Municípios deverão participar da formulação e execução da política de formação e desenvolvimento de RH para a saúde, bem como realizar pesquisas e estudos nesta área ${ }^{2}$.

A formação de RH para a saúde, diante do novo perfil epidemiológico e do modelo assistencial vigente no país, exige um reordenamento do processo de ensino-aprendizagem que supere as ideias flexrnerianas, que preconizam um modelo saúde-doença unicausal e biologicista, e faz pouca referência às dimensões social, psicológica e econômica da saúde ${ }^{3}$.

Outro fator importante que tem suscitado a reorientação da formação de RH para o SUS foi a implementação da Estratégia Saúde da Família (ESF), que demanda profissionais capacitados para novas práticas de trabalho integrais e interdisciplinares $^{4,5}$. Assim, as ações de promoção, proteção, prevenção, atenção precoce e reabilitação tornaram-se essenciais, sendo o perfil dos profissionais formados ainda inadequado para tal ${ }^{6}$.

Neste contexto, é necessário, portanto, reformular a formação profissional com base nos novos paradigmas e práticas assistenciais, buscando o engajamento do aluno na realidade local da população e dos serviços de saúde ${ }^{7,8}$.

Neste sentido, em 2001, o Ministério da Educação (MEC) deliberou novas Diretrizes Curriculares Nacionais (DCN) dos cursos de graduação da área de saúde, tornando-se clara a necessidade de se formar profissionais com ênfase na promoção, prevenção, recuperação e reabilitação da saúde, conforme preconizado nos princípios e diretrizes do SUS?.

Visando operacionalizar as DCN, os Ministérios da Saúde e da Educação propuseram estratégias como o Programa Nacional de Reorientação da Formação Profissional em Saúde (Pró-Saúde) e o Programa de Educação pelo Trabalho para a Saúde (PET-Saúde) ${ }^{10}$. Este último, objeto deste estudo, prevê a inserção precoce de estudantes da área da saúde na Atenção Primária à Saúde (APS), incentivando mudanças curriculares nos cursos de graduação, bem como a integração ensino-serviço, a educação permanente do profissional do serviço e o desenvolvimento de pesquisas nesse campo de atenção, visando sensibilizar e preparar os profissionais para o adequado enfrentamento das diferentes realidades de vida e saúde da população brasileira.

Em Belo Horizonte, Minas Gerais, foram instituídas parcerias entre as Universidades e a Secretaria Municipal de Saúde (SMSA-BH) para a execução do PET-Saúde no município, com destaque para a Universidade Federal de Minas Gerais (UFMG), que aderiu à proposta, em 2009, com treze grupos tutoriais abordando diferentes linhas de cuidado em saúde ${ }^{11}$. Em cada grupo havia a participação de um professor da UFMG, denominado tutor, profissionais da rede básica de saúde do município, os preceptores, e estudantes bolsistas e voluntários dos dez cursos de graduação da área da saúde da universidade.

Considerando que o PET-Saúde é uma estratégia que foi planejada para auxiliar o reordenamento da formação de RH para atender às demandas do SUS, é necessário avaliar tal programa em fase inicial de implantação visando aprimorar as atividades desenvolvidas e planejar ações futuras. Desse modo, este artigo objetivou analisar, sob a perspectiva dos alunos, a evolução do PET-Saúde UFMG/SMSA-BH no seu primeiro ano de implantação e sua contribuição para a formação profissional segundo as especificidades da Atenção Primária em Saúde.

\section{Metodologia}

Trata-se de estudo de coorte prospectivo envolvendo todos os alunos (bolsistas e voluntários) participantes do PET-Saúde UFMG/SMSA-BH, sendo excluídos os envolvidos nesta pesquisa.

Para a coleta de dados foi utilizado um questionário estruturado e autoaplicável, construído com base na Portaria Interministerial $n^{\circ} 1802 /$ 2008 e no projeto PET-Saúde/UFMG-SMSA$\mathrm{BH}^{12}$. O instrumento foi aplicado aos seis e dez meses de execução do programa e constou de questões sobre a caracterização do aluno, avaliação da metodologia e condições para o desenvolvimento das atividades do projeto.

A proposta da pesquisa foi inicialmente apresentada e discutida em conjunto com todos os tutores, que concordaram em envolver os monitores e voluntários nesta avaliação. Foi enviado para cada grupo tutorial um envelope contendo uma carta de apresentação da pesquisa, termo de consentimento livre e esclarecido e os questionários, em setembro de 2009 e em janeiro 2010, sendo recolhidos após o preenchimento. 
A análise quantitativa dos dados constou de análise descritiva e avaliação da distribuição das variáveis contínuas pela aplicação do teste de normalidade Kolmogorov-Smirnov. Adicionalmente, realizou-se a comparação entre as avaliações com aplicação dos testes estatísticos: QuiQuadrado de Pearson, Exato de Fisher e MannWhitney. Em todas as análises adotou-se um nível de significância de $5 \%$. As variáveis assimétricas foram apresentadas na forma de mediana $\mathrm{e}$ distribuição percentilar $\left(\mathrm{P}_{25}\right.$ e $\left.\mathrm{P}_{75}\right)$ e as demais em frequência. Para entrada dos dados utilizou-se o software estatístico Epi Info versão 6.0 e para análise, o Statistical Package for the Social Sciences (SPSS) versão 17.0.

O projeto foi aprovado pelo Comitê de Ética em Pesquisa da UFMG e os participantes assinaram o Termo de Consentimento Livre e Esclarecido.

\section{Resultados}

Do total de 170 participantes (120 bolsistas e 50 voluntários) do PET-Saúde/UFMG-SMSA/PBH, responderam ao primeiro questionário 129 indivíduos (75,9\%) e ao segundo 96 (56,5\%). Estes possuíam mediana de idade de 22 anos, sendo a maioria do sexo feminino, sem diferenças estatísticas entre as avaliações (Tabela 1).

Participaram da avaliação alunos das seguintes graduações: Educação Física, Farmácia, Fisioterapia, Gestão em Serviços de Saúde, Medicina Veterinária, Nutrição, Odontologia, Terapia Ocu- pacional, Enfermagem, Fonoaudiologia e Medicina, sendo esses dois últimos cursos os que tiveram o maior número de alunos envolvidos (13,2\% e $26,4 \%$, respectivamente na primeira avaliação e $10,1 \%$ e $20,9 \%$, respectivamente na segunda).

Todos os grupos tutoriais do PET-Saúde UFMG/SMSA-BH participaram da primeira avaliação $(n=13)$, mas na segunda dois grupos não retornaram os questionários preenchidos.

Os grupos desenvolveram ações nas seguintes linhas de pesquisa: Promoção de Modos de Vida Saudáveis; Saúde da Criança, do Idoso e da Mulher; e Interface Saúde e Ambiente (Tabela 1).

Em relação ao alcance dos objetivos propostos para o PET-Saúde, observou-se que a maioria dos alunos relatou que eles foram alcançados, tanto na primeira quanto na segunda avaliação, com destaque para o desenvolvimento de trabalho interdisciplinar e a produção acadêmica voltada para as necessidades do SUS. Por outro lado, o ponto de maior fragilidade foi a contribuição do PET para a reestruturação dos currículos dos cursos de graduação. Adicionalmente, verificou-se que na percepção dos alunos houve uma redução significativa $(\mathrm{p}=0,01)$ da capacitação do profissional em serviço da primeira para a segunda avaliação (Tabela 2).

Em relação aos temas trabalhados durante o processo de ensino-aprendizagem na primeira avaliação do PET-Saúde, verificou-se que a Atenção Primária à Saúde $(78,1 \%)$, Promoção da Saúde (77,3\%), Fluxos/dinâmica de Atendimento da Unidade Básica de Saúde (68,8\%) e Estratégia de

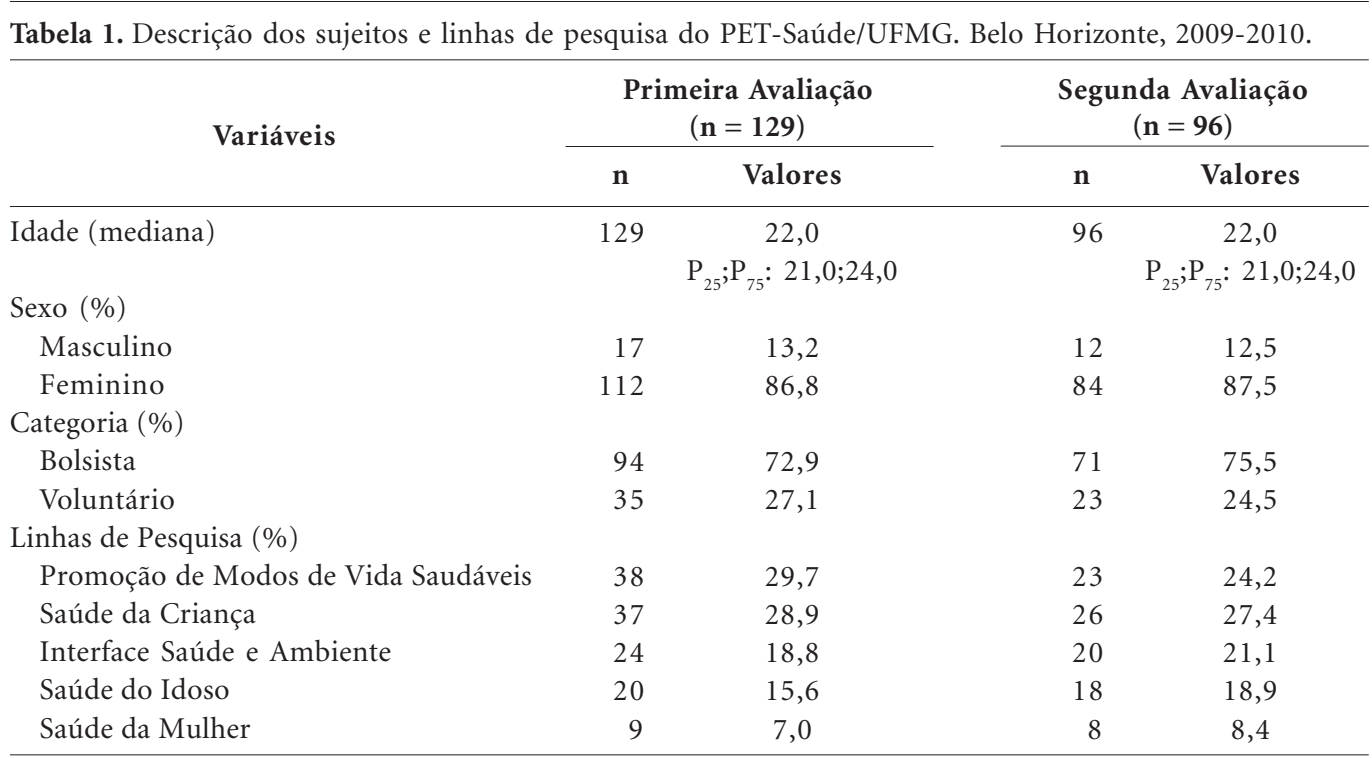


Saúde da Família $(64,8 \%)$ foram os mais abordados. Na segunda avaliação os aspectos mais trabalhados foram Atenção Primária à Saúde $(88,4 \%)$, Promoção da saúde $(81,1 \%)$, Metodologia Científica $(76,8 \%)$ e Fluxos/dinâmica de Atendimento da Unidade Básica de Saúde (UBS) $(71,6 \%)$. Além desses, foram também trabalhados outros temas como Saúde da Criança e do Idoso, Meio Ambiente e Saúde, Educação em Saúde, Integralidade da Assistência, Alimentação, Núcleo de Apoio à Saúde da Família (NASF), dentre outros.

De acordo com as duas avaliações, no processo de ensino-aprendizagem utilizaram-se preferencialmente como métodos a discussão em grupo $(88,1 \%$ vs. $92,6 \%)$, práticas inseridas no serviço de Atenção Primária à Saúde $(69,3 \%$ vs. $72,6 \%)$, leitura e fichamento de artigos (48,8\% vs. $71,6 \%)$ e aula expositiva $(48,0 \%$ vs. $43,2 \%)$. Também foram utilizados elaboração de materiais educativos, oficinas de trabalho e exibição de filmes, não havendo diferenças estatísticas entre os métodos.

Verificou-se que esses métodos favoreceram a aprendizagem para a maioria dos entrevistados nos dois momentos avaliados, oportunizando a integração ensino, pesquisa e extensão, e o trabalho interdisciplinar (Tabela 3). Segundo os entrevistados, o PET-Saúde contribuiu para a formação profissional preferencialmente nas áreas integradas de pesquisa, ensino e extensão $(27,1 \%)$, bem como de pesquisa $(40,6 \%)$, extensão $(33,3 \%)$ e ensino $(24,0 \%)$ isoladamente.

Em torno de 90,0\% dos alunos referiram que a interação e a comunicação estabelecidas entre tutor, preceptor e aluno favoreceram o processo ensino-aprendizagem, porém não houve alteração significativa entre a primeira e segunda avaliação, assim como para o incentivo permanente à participação, discussão e expressão livre das ideias (Tabela 3).

As atividades mais desenvolvidas no PETSaúde, segundo os alunos, foram pesquisa $(71,9 \%$ vs. $81,1 \%$ ), participação nas atividades das Equipes de Saúde da Família (76,2 vs. 76,6\%) e planejamento de ações $(68,1 \%$ vs. $66,0 \%)$. Por sua vez, as menos realizadas foram a interação com as organizações comunitárias, equipamentos sociais, conselhos locais de saúde e divulgação da pesquisa para a comunidade $(35,5 \%$ vs. $37,9 \%)$. Entretanto, houve aumento da interação com equipamentos sociais ao final do programa $(\mathrm{p}=$ 0,01) (Tabela 4).

No tocante à percepção do aluno acerca de sua capacidade para realizar as atividades propostas no PET-Saúde, não houve diferenças significativas. Destaca-se que na primeira avaliação os alunos se consideraram mais capacitados para as atividades relacionadas ao desenvolvimento de pesquisa $(92,3 \%)$, enquanto que na segunda avaliação houve maior destaque para a participação nas atividades das ESF (67,9\%) (Tabela 4).

Em relação às condições para o desenvolvimento das atividades do PET-Saúde, a Unidade Básica de Saúde como cenário de práticas foi avaliada como muito boa $(34,1 \%$ vs. $42,7 \%)$ e boa $(35,7 \%$ vs. $34,4 \%)$, sem diferenças significativas entre as avaliações. No entanto, a estrutura física foi considerada como adequada em parte $(50,8 \%$ vs. 51,0\%), devido principalmente à falta de espaço (salas) apropriado para o desenvolvimento das atividades (Figura 1).

Tabela 2. Objetivos alcançados durante a vivência no PET-Saúde/UFMG. Belo Horizonte, 2009-2010.

\begin{tabular}{|c|c|c|c|c|c|}
\hline \multirow{2}{*}{ Objetivos } & \multicolumn{2}{|c|}{$\begin{array}{l}\text { Primeira Avaliação } \\
\quad(\mathrm{n}=129)\end{array}$} & \multicolumn{2}{|c|}{$\begin{array}{l}\text { Segunda Avaliação } \\
(\mathrm{n}=96)\end{array}$} & \multirow{2}{*}{ Valor $\mathrm{p}$} \\
\hline & $\mathbf{n}$ & $\%$ & n & $\%$ & \\
\hline $\begin{array}{l}\text { Prática profissional dos estudantes na Atenção } \\
\text { Primária à Saúde }\end{array}$ & 62 & 48,4 & 50 & 52,1 & 0,26 \\
\hline Efetivação da integração ensino-serviço & 69 & 54,3 & 53 & 55,8 & 0,66 \\
\hline Fortalecimento da integração ensino-serviço & 79 & 62,2 & 58 & 61,1 & 0,27 \\
\hline Desenvolvimento de trabalho interdisciplinar & 103 & 80,5 & 79 & 82,3 & 0,17 \\
\hline $\begin{array}{l}\text { Realização de ações de promoção da saúde e } \\
\text { prevenção de agravos }\end{array}$ & 70 & 55,1 & 57 & 59,4 & 0,36 \\
\hline $\begin{array}{l}\text { Contribuição para reestruturação dos currículos dos } \\
\text { cursos de graduação }\end{array}$ & 49 & 38,3 & 38 & 40,0 & 0,79 \\
\hline $\begin{array}{l}\text { Produção acadêmica voltada para as necessidades } \\
\text { do Sistema Único de Saúde }\end{array}$ & 97 & 75,8 & 74 & 77,1 & 0,51 \\
\hline Capacitação profissional em serviço & 57 & 44,2 & 40 & 31,0 & 0,01 \\
\hline
\end{tabular}


Tabela 3. Avaliação dos métodos empregados pelo PET-Saúde/UFMG. Belo Horizonte, 2009-2010.

\begin{tabular}{|c|c|c|c|c|c|}
\hline \multirow{2}{*}{ Métodos } & \multicolumn{2}{|c|}{$\begin{array}{l}\text { Primeira Avaliação } \\
\quad(n=129)\end{array}$} & \multicolumn{2}{|c|}{$\begin{array}{l}\text { Segunda Avaliação } \\
(\mathrm{n}=96)\end{array}$} & \multirow{2}{*}{ Valor $\mathrm{p}$} \\
\hline & $\mathbf{n}$ & $\%$ & $\mathbf{n}$ & $\%$ & \\
\hline $\begin{array}{l}\text { Métodos utilizados favoreceram o ensino- } \\
\text { aprendizagem }\end{array}$ & 120 & 96,8 & 89 & 95,7 & 0,70 \\
\hline $\begin{array}{l}\text { Incentivo permanente à participação, discussão } \\
\text { e expressão livre das ideias }\end{array}$ & 112 & 89,6 & 78 & 85,7 & 0,49 \\
\hline $\begin{array}{l}\text { Metodologia empregada oportuniza o trabalho } \\
\text { interdisciplinar }\end{array}$ & 109 & 91,6 & 86 & 92,5 & 0,43 \\
\hline $\begin{array}{l}\text { Interação/comunicação estabelecida entre tutor, } \\
\text { preceptor e aluno favorece o processo ensino- } \\
\text { aprendizagem }\end{array}$ & 111 & 91,7 & 77 & 86,5 & 0,25 \\
\hline $\begin{array}{l}\text { Métodos adotados oportunizaram a integração } \\
\text { ensino, pesquisa e extensão }\end{array}$ & 113 & 93,4 & 80 & 90,9 & 0,59 \\
\hline
\end{tabular}

Tabela 4. Atividades desenvolvidas no período no PET-Saúde. Belo Horizonte, 2009-2010.

\begin{tabular}{|c|c|c|c|c|c|}
\hline \multirow[t]{2}{*}{ Atividades } & \multicolumn{2}{|c|}{$\begin{array}{l}\text { Primeira Avaliação } \\
(\mathrm{n}=129)\end{array}$} & \multicolumn{2}{|c|}{$\begin{array}{l}\text { Segunda Avaliação } \\
(\mathbf{n}=96)\end{array}$} & \multirow[t]{2}{*}{ Valor $\mathrm{p}$} \\
\hline & $\mathbf{n}$ & $\%$ & $\mathbf{n}$ & $\%$ & \\
\hline $\begin{array}{l}\text { Participação nas atividades das Equipes de } \\
\text { Saúde da Família }\end{array}$ & 93 & 76,2 & 72 & 76,6 & $0,39^{1}$ \\
\hline Capacitado para realizar & 84 & 90,3 & 53 & 67,9 & $0,89^{2}$ \\
\hline Planejamento de ações & 81 & 68,1 & 62 & 66,0 & $0,79^{1}$ \\
\hline Capacitado para realizar & 68 & 85,0 & 41 & 51,9 & $0,57^{2}$ \\
\hline Desenvolvimento de pesquisa & 87 & 71,9 & 77 & 81,1 & $0,38^{2}$ \\
\hline Capacitado para realizar & 72 & 92,3 & 43 & 53,1 & $0,76^{1}$ \\
\hline Capacitação de membros do PET-Saúde & 71 & 58,2 & 42 & 46,7 & $0,09^{1}$ \\
\hline Capacitado para realizar & 66 & 79,5 & 26 & 41,3 & $0,58^{2}$ \\
\hline Diagnóstico da área de abrangência da UBS & 53 & 44,2 & 41 & 43,6 & $0,75^{1}$ \\
\hline Capacitado para realizar & 43 & 62,3 & 42 & 59,2 & $0,41^{2}$ \\
\hline $\begin{array}{l}\text { Interação com organizações comunitárias, } \\
\text { equipamentos sociais, conselhos locais de saúde }\end{array}$ & 43 & 35,5 & 36 & 37,9 & $0,01^{1}$ \\
\hline Capacitado para realizar & 39 & 60,9 & 28 & 40,6 & $0,49^{2}$ \\
\hline Divulgação da pesquisa para a comunidade & 44 & 36,7 & 40 & 41,7 & $0,33^{1}$ \\
\hline Capacitado para realizar & 53 & 71,6 & 41 & 58,6 & $0,68^{2}$ \\
\hline $\begin{array}{l}\text { Divulgação da pesquisa em eventos e periódicos } \\
\text { científicos }\end{array}$ & 39 & 32,2 & 41 & 43,2 & 0,291 \\
\hline Capacitado para realizar & 40 & 58,8 & 25 & 39,1 & $0,35^{2}$ \\
\hline
\end{tabular}

1-Qui-quadrado; 2-Exato de Fisher.

De acordo com os alunos, as maiores dificuldades encontradas para a integração ensino-serviço foram a resistência dos profissionais da UBS não participantes do PET-Saúde (bolsistas: 31,9\% vs. $39,4 \%$; voluntários: $25,7 \%$ vs. $39,1 \%$ ), a comunicação (bolsistas: 24,5\% vs. 43,7\%; voluntários: $40,0 \%$ vs. $21,7 \%$ ) e a falta de capacitação pedagógica do preceptor para receber o aluno (bolsistas: 16,0\% vs. 43,7\%; voluntários: $14,3 \%$ vs. 21,7\%), sem diferenças significativas entre bolsistas e voluntários, bem com entre a primeira e a segunda avaliação.

Quanto ao desejo em participar do próximo PET-Saúde, verificou-se que inicialmente 56,4\% dos bolsistas e $57,1 \%$ dos voluntários relataram interesse, sendo que ao final foram $49,3 \%$ e $69,6 \%$, respectivamente. Entre os motivos para continuar no PET-Saúde destacaram a possibili- 
Primeira avaliação Segunda avaliação

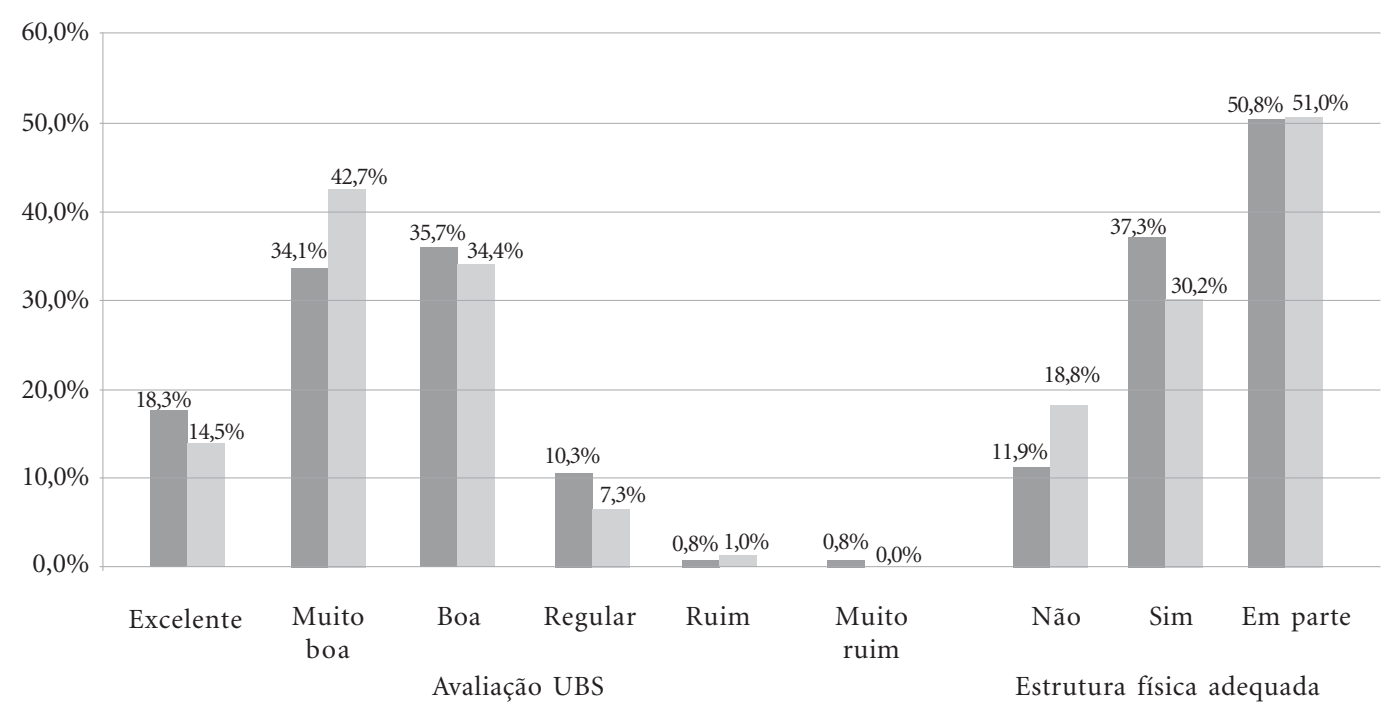

Figura 1. Avaliação da Unidade Básica de Saúde em quanto cenário para realização das atividades. Belo Horizonte, 2009-2010.

dade de crescimento e aprendizagem profissional e pessoal, e continuidade das atividades.

\section{Discussão}

O estudo revelou que, na perspectiva dos alunos, o PET-Saúde conseguiu alcançar em seu primeiro ano de execução, objetivos importantes como a integração ensino-serviço, a vivência da interdisciplinaridade no âmbito da APS, as práticas de promoção da saúde e prevenção de agravos, e o desenvolvimento de pesquisas direcionadas às necessidades do SUS. No entanto, como esperado, para um curto período de implantação, o programa não conseguiu impactar na reestruturação dos currículos dos cursos de graduação.

Ressalta-se que um processo de mudança curricular só acontece diante da inquietação dos atores e das exigências do mercado de trabalho ${ }^{13}$, o que demanda tempo e pressão dos envolvidos sobre a universidade. Assim, esse processo só será efetivado se os envolvidos estiverem inseridos cada vez mais precocemente na prática ${ }^{13}$, sendo o PETSaúde uma estratégia excepcional para isso.

Atualmente, a área de saúde requer profissionais capacitados para o enfrentamento dos prin- cipais problemas de saúde e das necessidades dos serviços ${ }^{13}$, cuja formação seja crítica, reflexiva, responsável e que contribua para o desenvolvimento social do país ${ }^{14}$. Os resultados do presente estudo mostram que os alunos perceberam o PET-Saúde como motivador da discussão e expressão livre das ideias entre os pares, além de incentivar a participação e a reflexão sobre as atividades desenvolvidas na APS.

Estudo realizado com alunos do curso de Medicina de uma escola pública de Ribeirão Preto, São Paulo, evidenciou que o estágio na Estratégia Saúde da Família contribuiu positivamente para a sua formação. Este fato se deu por ampliar seu olhar sobre o paciente, passando a considerar em sua avaliação e intervenção o contexto socioeconômico, emocional e cultural ${ }^{15}$. Ambos os estudos sinalizam para a importância da inclusão nos currículos da área de saúde de atividades teórico-práticas na Atenção Primária visando que o aluno vivencie em sua formação peculiaridades desse nível de atenção, como o trabalho em equipe, a longitudinalidade, a abordagem centrada na família e atuação na promoção e prevenção, e se empodere em relação à prática do cuidado integral ${ }^{16}$.

Destaca-se que o PET-Saúde, diferentemente do estágio profissional, não tem como foco o exer- 
cício das atribuições específicas de cada profissão no âmbito da APS, mas sim, das competências globais de um profissional da saúde. Desse modo, observou-se que menos da metade dos alunos, nas duas avaliações, consideraram que o programa contribuiu para a capacitação profissional em serviço. Ademais, houve uma redução significativa na segunda avaliação do percentual de alunos que consideraram que o PET-Saúde atingiu este objetivo. Os alunos em geral, estimulados pela sociedade e pela própria formação acadêmica, tendem a preferir atividades relacionadas aos processos de cura que envolvam a utilização de tecnologias duras ${ }^{17}$. Assim, como o PET incentiva o aluno a explorar o uso de tecnologias leves, ele torna-se, no ponto de vista do aluno, uma oportunidade menos hábil de capacitação profissional, apesar de esta ocorrer em sua prática.

Os alunos consideram que o PET-Saúde contribuiu para o fortalecimento da integração ensino-serviço, mas apontaram nas duas avaliações que a resistência dos profissionais da UBS não participantes do programa foi um grande empecilho para essa integração. Tal dificuldade não teve destaque no estudo realizado com alunos de $\mathrm{Me}$ dicina de uma escola pública sobre o estágio em Saúde da Família. Observou-se, pelo contrário, uma boa integração entre as Equipes de Saúde da Família e os alunos ${ }^{15}$. Cabe salientar que a dificuldade verificada aumentou com o passar do tempo, porém não de forma significativa. Acredita-se que essa resistência dos profissionais da UBS não participantes em acolher os alunos deva-se ao fato dos preceptores receberem incentivo financeiro do Ministério da Saúde para participarem do programa. Desse modo, evidencia-se a necessidade de melhor explicar o programa para os profissionais da UBS, bem como apresentar os critérios de seleção e as funções do preceptor visando superar o distanciamento dos profissionais da UBS em relação às atividades do PET-Saúde, potencializando assim as ações do aluno no serviço ${ }^{12}$.

Temas importantes para a formação do aluno para atuar junto à Estratégia de Saúde da Família, como Atenção Primária à Saúde, Promoção de saúde e Fluxos/dinâmica de Atendimento da UBS, tiveram uma maior abordagem no processo ensino-aprendizagem ao longo do programa. Essa abordagem é de suma importância, visto que o SUS tem exigido dos profissionais a clareza dos seus limites de atuação e intervenção, destacando o acolhimento do usuário e o entendimento do sistema como imprescindíveis para que consiga dar encaminhamento correto e resolutivo às demandas ${ }^{14}$.
Considerando que a formação de RH em saúde deve pautar-se na compreensão do modelo biopsicossocial e no trabalho interdisciplinar ${ }^{8}$, os métodos empregados no processo ensino-aprendizagem pelo PET-Saúde contribuíram sobremaneira para o exercício da interdisciplinaridade e da visão ampliada de saúde. Destaca-se que, para os alunos, oportunizar o trabalho interdisciplinar foi o objetivo que o programa conseguiu melhor atingir no período avaliado. Isso se deve a estruturação do PET-Saúde em grupos tutoriais compostos por alunos e profissionais de diferentes áreas do conhecimento, possibilitando a quebra das defesas corporativas e permitindo a troca e o aprendizado mútuos ${ }^{18}$, o que reforça a importância de cada profissional na construção da integralidade na atenção à saúde.

Vivenciar a interdisciplinaridade contribui de forma importante para o crescimento acadêmico e profissional do aluno, pois permite a compreensão integral do ser humano no contexto das relações sociais e do processo saúde-doença. Ademais, práticas interdisciplinares envolve flexibilização dos mandatos sociais e revisão das legislações profissionais, buscando uma nova profissionalização capaz de enfrentar desafios teórico-práticos na APS ${ }^{19}$.

Um aspecto importante para garantir uma maior efetividade das ações realizadas na APS é a articulação dos profissionais do SUS com a comunidade. No entanto, observa-se uma dificuldade dos profissionais da APS em realizarem ações extramuros. Na percepção dos alunos esta foi a atividade menos realizada no período avaliado, embora tenha apresentado alteração positiva entre as avaliações, demonstrando que ao longo do processo o aluno foi tendo maior contato com a comunidade, exercendo, ainda que de forma incipiente, algumas ações extramuros.

Essa participação na comunidade está em consonância com as recomendações do Ministério da Saúde, que ressalta a necessidade dos profissionais do SUS estimularem movimentos sociais e a organização comunitária, fortalecendo a participação popular, promovendo autonomia e empoderamento das comunidades na tomada de decisões sobre sua saúde ${ }^{20}$. Estudo realizado com alunos do curso de Odontologia de duas faculdades de Minas Gerais, em 2005, demonstrou a importância da vivência dos estudantes na Estratégia Saúde da Família, para aumentar sua sensibilidade ao vínculo que deve existir entre o profissional de saúde e a comunidade, o que poderá favorecer sua futura atividade profissional ${ }^{17}$. 
Os alunos ressaltaram que a maior contribuição do PET-Saúde para a sua formação profissional foi na área da pesquisa. O fato das atividades de pesquisa já estarem delineadas desde o início do programa e os tutores envolvidos no processo dominarem esse tipo de ação pode ter contribuído para que os alunos se sentissem mais bem preparados para a pesquisa em relação às atividades de ensino e extensão. Estas últimas foram sendo propostas ao longo do tempo a partir do conhecimento da unidade, da equipe de trabalho e de suas demandas, sem tanto domínio e apropriação de todos os envolvidos.

Destaca-se que a qualidade da produção universitária é orientada pela indissociabilidade entre ensino, pesquisa e extensão, responsável por promover uma consciência profissional eticamente fundamentada e empiricamente atualizada ${ }^{21}$. Desse modo, é necessário que o programa aprimore as ações no campo do ensino e extensão de modo a favorecer uma formação ainda mais completa do aluno. No entanto, é importante ressaltar que o PET-Saúde ao desenvolver pesquisa no âmbito da APS, contribuiu de forma relevante para a formação do aluno segundo os princípios do SUS destacados na Lei $8080^{2}$.

Um fator limitante apontado pelos alunos para o desenvolvimento ideal das atividades do programa foi a infraestrutura. A falta de espaço físico adequado para comportar profissionais, usuários, monitores e voluntários do PET-Saúde e alunos de outros programas, acabou por limitar o desenvolvimento de algumas atividades. No entanto, essa foi uma oportunidade para os alunos experimentarem o que os profissionais vivenciam em sua realidade, circunstância essa que exigiu deles maior pró-atividade e criatividade para vencer os obstáculos e tornar a UBS um espaço rico para o trabalho. Isso certamente contribui para a formação de um profissional diferenciado.

Diante disto, mais da metade dos monitores relataram que gostariam de continuar participando do PET-Saúde, corroborando o interesse dos alunos pelo programa, especialmente porque contribui para o desenvolvimento científico voltado para Atenção Primária, bem como desenvolve o senso de responsabilidade social nos estudantes ${ }^{11}$. Observou-se um percentual maior de voluntários e menor de bolsistas que demonstrou interesse em continuar no PET-Saúde. Isso pode ser explicado pelo fato dos bolsistas estarem em períodos mais avançados, e, portanto, almejarem outros estágios e pesquisas, enquanto os voluntários preferiam continuar no programa visando obter uma maior vivência de ensino, pesquisa e extensão no âmbito do SUS, e dar sequência à pesquisa que iniciaram.

Adicionalmente, tem-se a indisponibilidade de horário dentro da grade curricular dos alunos como fator limitante para continuidade no programa, dado que os bolsistas devem dedicar semanalmente oito horas às atividades presenciais e quatro horas fora do serviço, e os voluntários, quatro horas presenciais e quatro fora do serviço. Assim, é necessário pensar em mudanças curriculares que permitam maior disponibilidade de horários dos alunos para desenvolverem atividades dessa natureza.

Uma importante limitação deste estudo foi à inexistência de um sistema de informação sobre a participação dos alunos voluntários no programa, o que impediu a análise de viés de seleção, que permitiria conhecer o perfil dos alunos que não participaram da pesquisa. Atualmente, o programa conta com sistema de informação que abarca apenas os bolsistas, impedindo a comparação com os voluntários em relação ao perfil demográfico, características da graduação, tempo de permanência no programa ou em diferentes grupos tutoriais, linhas de pesquisa procuradas, entre outras características.

Ademais, como os questionários foram anônimos inviabilizando a realização de uma análise estatística pareada e identificação das perdas entre a primeira e segunda avaliação.

Formar recursos humanos com perfil adequado às necessidades sociais é um grande desafio e implica em propiciar aos alunos a capacidade de trabalho em equipe, comunicação e agilidade frente às situações a que estão expostos, como aqui verificado. A inserção dos alunos da área de saúde na Atenção Primária, por meio do PETSaúde, poderá possibilitar a vivência da interdisciplinaridade, longitudinalidade, atenção centrada na família, coordenação do cuidado, por meio de práticas integradas à comunidade, que por sua vez produzirão conhecimento e melhores serviços de saúde para a população. Desse modo, esse estudante se diferenciará pela formação críticoreflexiva e pela capacidade de aprender a aprender com a realidade em que se inserem. A avaliação dos alunos do PET-Saúde UFMG/SMSA-BH evidencia tais aspectos e sugere que esta constituiu uma estratégia positiva para a formação de RH em saúde, que atenda às necessidades do SUS. 


\section{Colaboradores}

ACM Pinto, IV Oliveira, ALS Santos, LES Silva e GSL Izidoro trabalharam na análise e interpretação dos dados, na redação do artigo e aprovou a versão a ser publicada. RD Mendonça realizou a análise e interpretação dos dados, revisão crítica e aprovou a versão a ser publicada. ACS Lopes foi responsável pela concepção e delineamento do estudo, revisão crítica e aprovou a versão a ser publicada.

\section{Agradecimentos}

Ao Ministério da Saúde pelas bolsas. A todos os participantes do PET-Saúde da Universidade Federal de Minas Gerais.

\section{Referências}

1. Brasil. Constituição da República Federativa do Brasil de 1988. Diário Oficial da União 1988; 5 out.

2. Brasil. Lei n. 8.080, de 19 de setembro de 1990. Dispõe sobre as condições para a promoção, proteção e recuperação da saúde, a organização e o funcionamento dos serviços correspondentes e dá outras providências. Diário Oficial da União 1990; 19 set.

3. Pagliosa FL, Da Ros MA. O relatório Flexner: para o bem e para o mal. Rev bras educ med 2008; 32(4):492-499.

4. Escorel S, Giovanella L, Mendonça MHM, Senna MCM. The Family Health Program and the construction of a new model for primary care in Brazil. Rev Panam Salud Publica 2007; 21(2-3):164-176.

5. Pires ROM. O pensamento crítico social de Paulo Freire sobre humanização e o contexto da formação do enfermeiro, do médico e do odontólogo [tese]. Ribeirão Preto (SP): Escola de Enfermagem de Ribeirão Preto, Universidade de São Paulo; 2008.

6. Gil CRR. Formação de recursos humanos em saúde da família: paradoxos e perspectivas. Cad Saude Publica 2005; 21(2):490-498.

7. Santana JP, Campos FE, Sena RR. Formação profissional em saúde: desafios para a universidade. Brasília: MS, OPS, UFRN; 1999.

8. Motta LB, Aguiar AC. Novas competências profissionais em saúde e o envelhecimento populacional brasileiro: integralidade, interdisciplinaridade e intersetorialidade. Cien Saude Colet 2007; 12(2):363372.

9. Moimaz SAS, Saliba NA, Zina LG, Saliba O, Garbin CAS. Práticas de ensino-aprendizagem com base em cenários reais. Interface (Botucatu) 2010; 14(32): 69-79.

10. Brasil. Ministérios da Educação e da Saúde. Portaria Interministerial no 1.802 , de 26 de agosto de 2008. Institui o Programa de Educação pelo Trabalho para a Saúde - PET - Saúde. Diário Oficial da União 2008; 27 ago.

11. Alves CR, Soares D, Menzel HJ, Cassiano J, Lemos S. Pet-Saúde. Universidade Federal de Minas Gerais Secretaria Municipal de Saúde de Belo Horizonte: uma experiência de integração ensino-pesquisa-extensão na atenção básica à saúde; $2010 \mathrm{Fev}$ 8-12; São Paulo, Brasil: PBL Congresso Internacional; 2010.

12. Caldas JB, Lopes ACS, Mendonça RD, Figueiredo A, Lonts JGA, Ferreira EF, Figueira TR. Percepção de Alunos quanto ao Programa de Educação pelo Trabalho para a Saúde (PET-Saúde). Rev Bras Educ Med 2012; 36(1 Supl. 2):33-41.

13. Freire RP, Guimarães RM, Henriques RLM, Mauro MYC. O Currículo Integrado da Faculdade de Enfermagem UERJ: uma reflexão sobre a Formação de Recursos Humanos para o SUS. Rev Bras Enferm 2003; 56(4):381-384.

14. Costa RKS, Miranda FAN. Formação profissional no SUS: oportunidades de mudanças na perspectiva da estratégia de saúde da família. Trab Educ Saude 2009; 6(3):503-517.

15. Campos MAF, Foster AC. Percepção e avaliação dos alunos do curso de medicina de uma escola médica pública sobre a importância do estágio em saúde da família na sua formação. Rev Bras Educ Med 2008; 32(1):83-89. 
16. Starfield B. Atenção Primária: equilíbrio entre necessidades de saúde, serviços e tecnologia. Brasília: UNESCO, Ministério da Saúde; 2002.

17. Sanchez HF, Drumond MM, Vilaça EL. Adequação de recursos humanos ao PSF: percepção de formandos de dois modelos de formação acadêmica em odontologia. Cien Saude Colet 2008; 13(2):523-531.

18. Vasconcelos EM. Complexidade e pesquisa interdisciplinar: epistemologia e metodologia operativa. Petrópolis: Vozes; 2002.

19. Martins de Sá JL. Gerontologia e interdisciplinaridade-fundamentos epistemológicos. Gerontologia 1998; 6(1):41-45.

20. Brasil. Ministério da Saúde(MS). Estratégias de promoção da alimentação saudável para o nível local. Relatório da oficina de Trabalho do I Seminário sobre Política Nacional de Promoção da Saúde. Brasília: MS; 2007.

21. Moita FMGSC, Andrade FCB. Ensino-pesquisa-extensão: um exercício indissociabilidade na pós-graduação. Rev Bras Educ 2009; 14(41):269-280.

Artigo apresentado em 02/03/2012

Aprovado em 13/05/2012

Versão final apresentada em 31/05/2012 